\title{
The XMM spectral catalog of SDSS optically selected Seyfert 2 galaxies
}

\author{
E. Koulouridis ${ }^{1,2}$, I. Georgantopoulos ${ }^{1}$, G. Loukaidou $^{3}$, A. Corral ${ }^{1}$, A. Akylas ${ }^{1}$, L. Koutoulidis ${ }^{1}$, \\ E. F. Jiménez-Andrade ${ }^{4,5}$, J. León-Tavares ${ }^{5,6}$, and P. Ranalli ${ }^{1,7}$ \\ ${ }^{1}$ Institute for Astronomy \& Astrophysics, Space Applications \& Remote Sensing, National Observatory of Athens, \\ 15236 Palaia Penteli, Greece \\ e-mail: elias.koulouridis@cea.fr \\ 2 Service d'Astrophysique AIM, CEA Saclay, 91191 Gif-sur-Yvette, France \\ 3 Faculty of Physics, School of Sciences, Univ. of Athens, Panepistimiopolis, 15771 Ilissia, Greece \\ 4 Argelander-Institut für Astronomie, Universität Bonn, Auf dem Hügel 71, 53121 Bonn, Germany \\ 5 Instituto Nacional de Astrofísica Óptica y Electrónica (INAOE), Apartado Postal 51 y 216, 72000 Puebla, Mexico \\ ${ }^{6}$ Sterrenkundig Observatorium, Universiteit Gent, Krijgslaan 281-S9, 9000 Gent, Belgium \\ 7 Lund Observatory, Box 43, 22100 Lund, Sweden
}

Received 12 May 2015 / Accepted 5 November 2015

\section{ABSTRACT}

\begin{abstract}
We present an X-ray spectroscopic study of optically selected (SDSS) Seyfert 2 (Sy2) galaxies. The goal is to study the obscuration of Sy2 galaxies beyond the local universe, using good quality X-ray spectra in combination with high S/N optical spectra for their robust classification. We analyzed all available XMM-Newton archival observations of narrow emission line galaxies that meet the above criteria in the redshift range $0.05<z<0.35$. We initially selected narrow line AGN using the SDSS optical spectra and the BPT classification diagram. We further modeled and removed the stellar continuum, and we analyzed the residual emission line spectrum to exclude any possible intermediate-type Seyferts. Our final catalog comprises 31 Sy2 galaxies with median redshift $z \sim 0.1$. X-ray spectroscopy is performed using the available X-ray spectra from the 3XMM and the XMMFITCAT catalogs. Implementing various indicators of obscuration, we find seven ( 23\%) Compton-thick AGN. The X-ray spectroscopic Compton-thick classification agrees with other commonly used diagnostics, such as the X-ray to mid-IR luminosity ratio and the X-ray to [OIII] $\lambda 5007$ luminosity ratio. Most importantly, we find four $(\sim 13 \%)$ unobscured Sy2 galaxies, at odds with the simplest unification model. Their accretion rates are significantly lower than the rest of our Sy2 sample, in agreement with previous studies that predict the absence of the broad line region below a certain Eddington ratio threshold.
\end{abstract}

Key words. galaxies: active - galaxies: Seyfert $-\mathrm{X}$-rays: galaxies $-\mathrm{X}$-rays: general - surveys

\section{Introduction}

Nearly thirty years ago, the first discovery by Miller \& Antonucci (1983) of broad permitted emission lines and a clearly non-stellar continuum in the polarized spectrum of the archetypal Seyfert 2 (Sy2), NGC 1068, was just the beginning of numerous similar observations in a wide variety of galaxies. Ten years later, the unification model of active galactic nuclei (AGN) was formulated upon these observations (Antonucci 1993). According to the unification model, all AGN are intrinsically identical, while the only cause of their different observational features is the orientation of an obscuring torus with respect to our line of sight. In more detail, the AGN type depends on the obscuration of the broad line region (BLR), a small area at close proximity to the SMBH where the broad permitted lines are produced. If the torus happens to be between the observer and the BLR, the optical emission and even the soft $\mathrm{X}$-rays are absorbed. Optical spectropolarimetric observations can reveal the hidden broad line region (HBLR) by highlighting its scattered emission. The observed narrow permitted emission lines are produced at far larger distances from the core, where the torus is irrelevant. As a prediction of this model, the presence and strength of the broad optical emission lines, hence the derived optical spectral type (from type 1 AGN/Sy1s to type $2 \mathrm{AGN} / \mathrm{Sy} 2 \mathrm{~s}$, and the intermediate types), should correlate with the amount of intervening material as measured in X-rays.

X-ray observations can reveal the exact density of the obscuring torus, even for mildly obscured sources. X-ray surveys with Ginga (Smith \& Done 1996) and ASCA (Turner et al. 1997) measured column densities between $10^{22}$ and up to a few times $10^{24} \mathrm{~cm}^{-2}$ in type $2 \mathrm{AGN}$ samples. More recently, Akylas \& Georgantopoulos (2009) and Brightman \& Nandra (2011), using XMM-Newton, and Jia et al. (2013, JJ13 hereafter), using Chandra, also studied the obscuration of type $2 \mathrm{X}$-ray sources in detail (see also Brandt \& Alexander 2015 for a recent review). However, even in the hard X-ray band, the X-ray surveys may be missing a fraction of highly obscured sources. These sources are called Compton-thick AGN (see reviews by Comastri 2004 and Georgantopoulos 2013), and they present very high obscuring column densities $\left(>10^{24} \mathrm{~cm}^{-2}\right.$, corresponding to an optical reddening of $\left.A_{V}>100\right)$. Even though Compton-thick AGN are abundant in the optically selected samples of nearby Seyferts (e.g., Risaliti et al. 1999), only a few tens of Comptonthick sources have been identified from X-ray data. Moreover, Krumpe et al. (2008) found no Compton-thick QSO in their high redshift $(z>0.5)$, X-ray selected sample, implying a possible redshift evolution, though this may be due to selection. 
Although the population of Compton-thick sources remains elusive, there is concrete evidence of its presence. The X-ray background (XRB) synthesis models can explain the peak of the X-ray background at 30-40 keV, where most of its energy density lies, (Frontera et al. 2007; Churazov et al. 2007) only by invoking a large number of Compton-thick AGN (Gilli et al. 2007). We note, however, that other models (e.g., Treister et al. 2009; Akylas et al. 2012) succeed in explaining the XRB spectrum assuming a lower fraction of CT sources. Additional evidence of a Compton-thick population comes from the directly measured space density of black holes in the local Universe. It is found that this space density could be up to a factor of two higher than predicted from the X-ray luminosity function (Marconi et al. 2004). This immediately suggests that the X-ray luminosity function is missing an appreciable number of obscured AGN.

On the other hand, although widely accepted today, the unification model cannot explain a series of observations. For example, Tran et al. (2001) noticed the absence of a HBLR in polarized light in many Sy2 galaxies (non-HBLR Sy2 galaxies), suggesting that there is a class of true Sy2 galaxies that intrinsically lack the BLR (see Ho 2008, for a review). Theoretical models attributed the absence of a BLR to either a low Eddington ratio (Nicastro 2000) or to low luminosity (Elitzur \& Shlossman 2006). Many studies propose an evolutionary model where a fraction of Sy2 represents the first or the last phase in the life of an AGN (Hunt \& Malkan 1999; Dultzin-Hacyan 1999; Krongold et al. 2002; Levenson et al. 2001; Koulouridis et al. 2006a,b, 2013; Koulouridis 2014; Elitzur et al. 2014). This was supported by studies of the local environment of Seyfert galaxies, which showed that Sy2s reside in richer environments compared to Sy1s (e.g., Villaroel \& Korn 2014). Unobscured low-luminosity Sy2s were detected via investigation of their X-ray properties (e.g., Pappa et al. 2000; Panessa \& Bassani 2002; Akylas \& Georgantopoulos 2009). Models of galaxy formation also support this scenario: for example, Hopkins et al. (2008) assert that the AGN is heavily obscured during its birth. During the buildup of its black hole mass, it blows away its cocoon, becoming an unobscured AGN.

In this paper, we compile a sample of bona fide optically selected Sy2 galaxies using the SDSS spectra from the data release 10 (DR10). We cross-correlate our sample with the 3XMM/XMMFITCAT spectral catalog (Corral et al. 2015), which contains good quality spectra (at least 50 net counts per XMM detector). We identify a sample of 31 Sy2 galaxies with available X-ray spectra in the redshift range $z=0.05-0.3$. Our study is complemented by X-ray, mid-IR, and [OIII] luminosity ratio diagnostics (Georgantopoulos et al. 2013; Trouille $\&$ Barger 2010). This study provides an extension of previous X-ray studies in the local Universe (e.g., Akylas \& Georgantopoulos 2009) but also of similar studies at higher redshifts (e.g., JJ13) because of the high signal-to-noise ratio $(\mathrm{S} / \mathrm{N})$ $\mathrm{X}$-ray spectra used.

We describe our sample selection in Sect. 2, the X-ray analysis in Sect. 3, while our results and conclusions are presented in Sects. 4 and 5, respectively. Throughout this paper we use $H_{0}=72 \mathrm{~km} \mathrm{~s}^{-1} \mathrm{Mpc}^{-1}, \Omega_{\mathrm{m}}=0.27$, and $\Omega_{\Lambda}=0.73$.

\section{Sample selection}

Our sample is composed of Seyfert 2 galaxies with available $\mathrm{X}$-ray spectra within the XMM-Newton Serendipitous Source catalog (Watson et al. 2009; Rosen et al. 2015) and optical spectra within the SDSS-DR10. The names of the sources are taken from the SDSS database. Also, a sequence number is given to each source in the current paper (see Table 1). In the diagrams, interesting sources are followed by their sequence numbers. In the text, the names are followed by the sequence number in parenthesis to make it easier for the reader to trace the sources in the tables and the diagrams.

\subsection{X-ray selection}

The XMM-Newton catalog is the largest catalog of X-ray sources ever built. Its current version, $3 \mathrm{XMM}-\mathrm{DR} 4^{1}$, contains photometric information for half a million source detections, and in addition, spectral and timing data for $\sim 120000$ of them. The count limit adopted by the $3 \mathrm{XMM}-\mathrm{DR} 4$ pipeline to derive spectral products is of 100 EPIC net (background subtracted) counts, in order to allow reliable X-ray spectral extraction and analysis.

The starting sample was extracted from the XMM-Newton/ SDSS-DR7 cross-correlation presented in Georgakakis \& Nandra (2011), including more than 40000 X-ray sources. We first selected the sources detected in the X-ray hard band $(2-8 \mathrm{keV})$, a band less affected by obscuration than is the soft one $(0.5-2 \mathrm{keV})$. A total of 1275 sources were found to have available optical spectra within SDSS-DR7. Out of these, 1018 sources had available 3XMM-DR4 spectral data. The corresponding SDSS optical spectra of these 1018 sources were manually examined in order to identify Seyfert 2 galaxies, resulting in our final sample of Sy2s (see next section). It is worth noting that two of these sources have more than one XMM-Newton observation with spectra within 3XMM-DR4, from which we used the longest one.

\subsection{Optical selection}

We built the final Sy2 sample based on the emission line properties of their SDSS optical spectra. Initially, we selected only emission line galaxies with redshifts between $z=0.05$ and $z=0.35$. The lower redshift limit excludes all already extensively studied and well-known Seyferts (e.g., Akylas \& Georgantopoulos 2009), while the upper limit ensures that the $\mathrm{H} \alpha$ and [NII] emission lines are within the SDSS spectral range. Furthermore, we excluded all objects where the velocity dispersion of the $\mathrm{H} \alpha$ line is greater than $500 \mathrm{~km} \mathrm{~s}^{-1}$, since these objects are certainly broadline AGN. The rest of the objects were placed on a BPT diagram (Baldwin et al. 1981) and star-forming galaxies, composite galaxies, and LINERS were removed according to the criteria of Kewley et al. (2001) and Schawinski et al. (2007).

We used the MPA-JHU emission line fluxes published in DR8 (Brinchmann et al. 2004; Tremonti et al. 2004), although DR10 also contains data from the recent spectroscopic analysis of the Portsmouth Group (Thomas et al. 2013). However, the latter includes only those galaxies from the first two years of observations of the SDSS-III/Baryonic Oscillation Spectroscopic Survey (BOSS) collaboration. We note that a comparison between the two databases by Thomas et al. (2013) has shown that the discrepancy between the calculated emission line fluxes is small. However, the comparison was made after rescaling the Portsmouth values with a factor provided by the "spectofiber" keyword in the MPA-JHU database. This rescaling was originally applied to the MPA-JHU data so that the synthetic $r$-band magnitude computed from the spectrum matches the $r$-band fiber magnitude measured by the photometric pipeline. The use of

http://xmmssc-wWw.star.le.ac.uk/Catalogue/3XMM-DR4/ 
Table 1. X-ray observations.

\begin{tabular}{|c|c|c|c|c|c|c|c|c|}
\hline $\begin{array}{l}N \\
(1)\end{array}$ & $\begin{array}{l}\text { Name } \\
(2)\end{array}$ & $\begin{array}{c}\text { Obsid } \\
\text { (3) }\end{array}$ & $\begin{array}{l}\text { RA } \\
(4)\end{array}$ & $\begin{array}{c}\text { Dec } \\
(5)\end{array}$ & $\begin{array}{c}z \\
(6) \\
\end{array}$ & $\begin{array}{c}N_{\mathrm{H}}\left(\times 10^{22}\right) \\
(7)\end{array}$ & $\begin{array}{c}\text { Exposure time } \\
(8)\end{array}$ & $\begin{array}{l}\text { Counts } \\
(9)\end{array}$ \\
\hline 1 & J080429.14+235444.1 & 504102101 & 121.1219 & 23.9127 & 0.07432 & 3.18 & $18300 /-/-$ & $86 / 0 / 0$ \\
\hline 2 & J080535.00+240950.3 & 203280201 & 121.3961 & 24.1645 & 0.05971 & 10 & $5598 / 8$ & $125 / 83 / 0$ \\
\hline 3 & J083139.08+524205.6 & 92800201 & 127.9131 & 52.7016 & 0.05855 & 1.16 & $60280 / 70920 / 71790$ & $334 / 127 / 166$ \\
\hline 4 & J084002.36+294902.6 & 504120101 & 130.0095 & 29.8175 & 0.06481 & 1.83 & $17870 / 22630 / 22640$ & $1072 / 424 / 428$ \\
\hline 5 & J085331.05+175339.0 & 305480301 & 133.3791 & 17.8942 & 0.18659 & 2.75 & $34560 /-/-$ & $173 / 0 / 0$ \\
\hline 6 & J091636.53+301749.3 & 150620301 & 139.1524 & 30.2969 & 0.12339 & 1.2 & $9049 / 9392 /-$ & $431 / 151 / 0$ \\
\hline 7 & $\mathrm{~J} 100129.41+013633.8$ & 302351001 & 150.3724 & 1.6095 & 0.10423 & $3 .($ & $1650 / 42310 / 42540$ & $310 / 98 / 127$ \\
\hline 8 & J101830.79+000504.9 & 402781401 & 154.6286 & 0.084 & 0.06233 & 3 & $15700 / 20540 / 20600$ & $753 / 398 / 397$ \\
\hline 9 & $\mathrm{~J} 103408.58+600152.1$ & 306050701 & 158.5360 & 60.0307 & 0.05101 & 1 & 8311 & 133 \\
\hline 10 & $\mathrm{~J} 103456.37+393941.0$ & 506440101 & 158.7349 & 3960 & 0 & 1.9 & $68400 / 830$ & $7 / 120$ \\
\hline 11 & $\mathrm{~J} 103$ & 506440101 & 158.8154 & 39. & 10 & & $-/ 83$ & $0 / 1$ \\
\hline 12 & 53.8 & 405240901 & 161.1109 & & 0.20991 & & $\mid-1-$ & \\
\hline 13 & $\mathrm{~J} 11$ & 107860201 & 170. & 43. & 0 & & & \\
\hline 14 & $\mathrm{~J} 11$ & 5041 & & & 0 & & $17490 /$ & /127 \\
\hline 15 & $\mathrm{~J} 114$ & 2042 & 177.1 & 53. & 0. & 1.23 & 17 & 11 \\
\hline 16 & J1218 & 400560301 & 184.6649 & 47.1 & $0 .($ & 1.00 & 37570 & $0 / 8$ \\
\hline 17 & J123056.11+155212.2 & 112552101 & 187.4978 & 13.5183 & 0.09816 & 2.3 & $8394 /-/-$ & $80 / 0 / 0$ \\
\hline 18 & $\mathrm{~J} 122959.45+133105.7$ & 106061001 & 187.7338 & 15.87 & 0.18768 & 2.0 & $4660 /-/ 8979$ & $96 / 0 / 98$ \\
\hline 19 & $\mathrm{~J} 124214.47+141147.0$ & 504240101 & 190.5607 & 14.196 & 0.15710 & 2.2 & $59590 /-/ 80240$ & $1465 / 0 / 665$ \\
\hline 20 & $\mathrm{~J} 125743.06+273628.2$ & 124710201 & 194.4296 & 27.608 & 0.06839 & 1 & $30010 /-/-$ & $119 / 0 / 0$ \\
\hline 21 & $\mathrm{~J} 130920.52+212642.7$ & 163560101 & 197.3359 & 21.4453 & 0.27858 & 1.5 & $-/ 28390 / 28700$ & $0 / 191 / 215$ \\
\hline 22 & $\mathrm{~J} 131104.66+272807.2$ & 21740201 & 197.7694 & 27.469 & 0.23975 & 2. & $35000 / 43000 / 43100$ & $267 / 73 / 75$ \\
\hline 23 & $\mathrm{~J} 132525.63+073607.5$ & 200730201 & 201.3567 & 7.6022 & 0.12402 & $5 .($ & $26900 /-/-$ & $174 / 0 / 0$ \\
\hline 24 & J134245.85+403913.6 & 70340701 & 205.6908 & 40.6537 & 0.08926 & 1.5. & $26010 / 35340 / 35100$ & $393 / 220 / 216$ \\
\hline 25 & $\mathrm{~J} 135436.29+051524.5$ & 404240101 & 208.6515 & 5.2564 & 0.08152 & $7 .($ & $11020 /-/ 15780$ & $168 / 0 / 101$ \\
\hline 26 & $\mathrm{~J} 141602.13+360923.2$ & 14862010 & 214.0089 & 36.1567 & 0.17100 & 2.5 & 10910/15780/16140 & 271/146/145 \\
\hline 27 & $\mathrm{~J} 145^{\circ}$ & 502 & 2 & & & 11.4 & $-1-$ & $71 / 0 / 0$ \\
\hline 28 & 05.0 & 3057 & 226.8330 & 0.4847 & 0.18219 & & 9931/-/- & $227 / 0 / 0$ \\
\hline 29 & $.38+010816.8$ & 402781001 & 226.9764 & 1.1381 & 0.06099 & 9.81 & $0 / 17900 / 1^{\prime}$ & $222 / 81 / 81$ \\
\hline 30 & J215649.51-074532.4 & 654440101 & 329.2059 & -7.7589 & 0.05541 & 5.22 & $42310 / 73600 / 75600$ & $134 / 63 / 56$ \\
\hline 31 & J224323.18-093105.8 & 503490201 & 340.8464 & -9.5185 & 0.14509 & 2.72 & $-/ 113700 / 114500$ & $0 / 246 / 247$ \\
\hline
\end{tabular}

Notes. (1) sequence number; (2) SDSS name; (3) XMM-Newton observation ID number; (4) spectroscopic redshift; (5) right ascension; (6) declination; (7) galactic column density in atoms $/ \mathrm{cm}^{2}$; (8) exposure time for the PN/MOS1/MOS2 detectors in seconds; (9) counts on the PN/MOS1/MOS2 detectors.

either database does not significantly affect the BPT diagram, since we only need the emission line ratios.

We note that in some cases the broadening of the Balmer lines cannot be automatically detected (Seyfert 1.5, 1.8, and especially 1.9), since it only affects the lower part of the lines. As a result, the automated modeling of the line by a single Gaussian may result in lower velocity dispersion values than what is expected from a broad line profile, and the source may be misclassified as a narrow-line AGN. However, since we sought a broad-line-free sample, the spectra of all remaining AGN were eye-inspected with the "interactive spectrum" tool of the SDSS, and all evident intermediate-type Seyferts were removed. After the above filtering, the catalog of Sy2s included 40 objects.

Despite the above selection, a number of sources in our sample still have discrepant classifications in the literature; i.e., eight of the sources are listed as Sy1s in Véron-Cetty \& Véron (2010) catalog, plus another one in the NED (NASA extragalactic database). Although none of these objects can actually be a Sy1, we proceeded with our own optical spectrum analysis to determine whether there is any broadening of the permitted emission lines.

\subsection{Optical spectrum analysis}

The spectra have been retrieved from the SDSS-DR10 and corrected for Galactic extinction using the maps of Schlegel (1998).
We use the stellar population synthesis code STARLIGHT ${ }^{2}$ to obtain the best fit to an observed spectrum $O_{\lambda}$, taking the corresponding flux error into account. The best fit is a combination of single stellar populations (SSP) from the evolutionary synthesis models of (Bruzual 2003) and a set of power laws to represent the AGN continuum emission. Following the latter approach, several studies have been successful at disentangling the host galaxy and AGN emission components in SDSS spectra (Cid-Fernandes 2011; León-Tavares et al. 2011).

We use a base of $150 \mathrm{SSP}$ plus six power laws in the form $F(\lambda)=10^{20}(\lambda / 4020)^{\beta}$, where $\beta=-0.5,-1,-1.5,-2,-2.5,-3$. Each SSP spans six metallicities, $Z=0.005,0.02,0.2,0.4,1$, and $2.5, Z_{\odot}$, with 25 different ages between 1 Myr and $18 \mathrm{Gyr}$. Extinction in the galaxy is taken into account in the synthesis, assuming that it arises from a foreground screen with the extinction law of (Cardelli 1989). The code finds the minimum $\chi^{2}$,

$\chi^{2}=\sum_{\lambda}\left(\frac{O_{\lambda}-M_{\lambda}}{\sigma_{\mathrm{obs}}}\right)$,

where $M_{\lambda}$ is the model spectrum (SSP and power laws), obtaining the corresponding physical parameters of the modeled spectrum: star formation history, $x_{j}$, as a function of a base of SSP models normalized at $\lambda_{0}, b_{j, \lambda}$, extinction coefficient of predefined extinction laws, $r_{\lambda}$, and velocity dispersion $\sigma_{\star}$, which

2 http://www.starlight.ufsc.br/ 
Table 2. X-ray spectral analysis.

\begin{tabular}{|c|c|c|c|c|c|c|c|c|c|c|c|c|}
\hline (1) & $\begin{array}{c}N_{\mathrm{H}} \\
\left(\times 10^{22}\right) \\
(2)\end{array}$ & $\begin{array}{l}\Gamma_{\text {soft }} \\
(3)\end{array}$ & $\begin{array}{c}\Gamma_{\text {hard }} \\
\text { (4) }\end{array}$ & $E W$ & $\begin{array}{c}\text { Flux } \\
\left(\times 10^{-14}\right) \\
(6)\end{array}$ & $\begin{array}{c}L_{\mathrm{X}} \\
\left(\times 10^{43}\right) \\
(7)\end{array}$ & $\mathrm{p} 1 / \mathrm{p} 2$ & $\begin{array}{l}\text { cstat/d.o.f. } \\
\text { (9) }\end{array}$ & $\begin{array}{c}L_{[\mathrm{OIII}]} \\
\left(\times 10^{42}\right) \\
(10)\end{array}$ & $\begin{array}{c}L_{12} \\
\left(\times 10^{43}\right) \\
(11)\end{array}$ & $\begin{array}{c}L_{\mathrm{bol}} \\
\left(\times 10^{43}\right) \\
(12)\end{array}$ & $\log \left(M_{\mathrm{BH}}\right)$ \\
\hline 1 & $18.84_{-5.56}^{+7.68}$ & $7.6_{-4.6}^{+7.6}$ & $1.8^{\ddagger}$ & $<0.33$ & 22.2 & 0.27 & 0.001 & $105.2 / 96$ & 0.18 & 0.4 & 1.1 & 6.9 \\
\hline 2 & $45.83_{-13.94}^{+21.30}$ & $2.4_{-0.4}^{+0.4}$ & $1.8^{\ddagger}$ & $0.29_{-0.24}^{+0.71}$ & 25.0 & 0.20 & 0.015 & $165.25 / 210$ & 0.60 & 0.2 & 0.3 & 6.8 \\
\hline 3 & $23.56_{-3.79}^{+4.00}$ & $2.1_{-0.4}^{+0.4}$ & $2.1_{-0.4}^{+0.4}$ & $0.26_{-0.13}^{+0.20}$ & 9.3 & 0.07 & 0.012 & $537.63 / 618$ & 0.17 & 0.7 & 1.0 & 6.3 \\
\hline 4 & $54.24_{-5.82}^{+5.32}$ & $2.8_{-0.1}^{+0.2}$ & $1.4_{-0.7}^{+0.7}$ & $0.30_{-0.80}^{+0.80}$ & 69.7 & 0.63 & 0.024 & $1088.68 / 1403$ & 1.11 & 8.8 & 19.9 & 7.6 \\
\hline 5 & $26.38_{-20.54}^{+27.12}$ & $2.5_{-0.7}^{+0.7}$ & $1.8^{\ddagger}$ & $<0.17$ & 17.8 & 1.20 & 0.293 & $147.19 / 189$ & 5.77 & 11.4 & 25.9 & 7.9 \\
\hline 6 & $<0.06$ & \multicolumn{2}{|c|}{$1.8_{-0.1}^{+0.2}$} & $<0.74$ & 22.4 & 0.87 & & $425.68 / 522$ & 3.47 & 1.8 & 2.8 & 8.2 \\
\hline 7 & $4.75_{-1.40}^{+1.28}$ & $1.4_{-0.4}^{+0.4}$ & $1.4_{-0.4}^{+0.4}$ & $0.29_{-0.17}^{+0.20}$ & 15.6 & 0.39 & 0.018 & $465.96 / 529$ & 0.07 & 1.4 & 1.9 & 7.2 \\
\hline 8 & $2.34_{-0.32}^{+0.38}$ & \multicolumn{2}{|c|}{$1.5_{-0.2}^{+0.2}$} & $<0.10$ & 60.1 & 0.54 & & $825.83 / 1078$ & 0.04 & 0.3 & 0.4 & 6.2 \\
\hline $9^{\dagger}$ & $30.32_{-11.62}^{+19.24}$ & $2.9_{-0.2}^{+0.2}$ & $1.8^{\ddagger}$ & $1.30_{-0.48}^{+0.80}$ & 17.8 & 0.07 & 0.293 & $430.95 / 474$ & 4.98 & 7.4 & 10.3 & 8.2 \\
\hline 10 & $64.58_{-23.33}^{+29.99}$ & $3.0_{-0.2}^{+0.2}$ & $1.8^{\ddagger}$ & $0.52_{-0.25}^{+0.26}$ & 4.1 & 0.20 & 0.102 & $505.46 / 546$ & 5.37 & 4.4 & 6.2 & 8.2 \\
\hline 11 & $14.35_{-5.85}^{+6.61}$ & $6.3_{-1.5}^{+2.1}$ & $2.0_{-1.1}^{+1.2}$ & $<1.21$ & 9.6 & 0.25 & 0.007 & $189.43 / 210$ & 0.22 & 7.4 & 16.7 & 7.6 \\
\hline 12 & $90.26_{-48.60}^{+31.61}$ & $1.5_{-0.9}^{+0.9}$ & $1.5_{-0.9}^{+0.9}$ & $<13.68$ & 11.6 & 0.92 & 0.004 & $82.28 / 98$ & 1.90 & 87.9 & 118.0 & 8.7 \\
\hline 13 & $5.49_{-2.51}^{+2.68}$ & $1.3_{-0.7}^{+0.6}$ & $1.3_{-0.7}^{+0.6}$ & $0.32_{-0.28}^{+0.85}$ & 19.1 & 0.95 & 0.054 & $140.57 / 186$ & 0.23 & 2.8 & 6.3 & 6.0 \\
\hline 14 & $130.46_{-56.34}^{+69.32}$ & $2.9_{-0.2}^{+0.2}$ & $1.8^{\ddagger}$ & $<0.65$ & 7.6 & 0.04 & 0.006 & $434.45 / 462$ & 6.62 & 22.2 & 50.8 & 7.6 \\
\hline 15 & $1.92_{-0.69}^{+0.88}$ & \multicolumn{2}{|c|}{$1.8_{-0.5}^{+0.6}$} & $<0.48$ & 144 & 3.37 & & $169.96 / 204$ & 0.64 & 0.9 & 1.2 & 7.3 \\
\hline $16^{\dagger}$ & $16.07_{-10.34}^{+39.22}$ & $2.9_{-0.4}^{+0.4}$ & $1.8^{\ddagger}$ & $0.85_{-0.66}^{+0.75}$ & 7.9 & 0.16 & 0.173 & $145.04 / 155$ & 5.47 & 9.4 & 13.1 & 7.3 \\
\hline 17 & $4.83_{-2.48}^{+2.80}$ & $2.3_{-1.1}^{+1.0}$ & $2.3_{-1.1}^{+1.0}$ & $\star$ & 12.6 & 0.30 & 0.015 & $82.11 / 85$ & 2.06 & 4.8 & 6.3 & 7.4 \\
\hline 18 & $1.81_{-0.87}^{+1.08}$ & \multicolumn{2}{|c|}{$1.2_{-0.5}^{+0.5}$} & $<0.35$ & 45.2 & 3.81 & & $183.1 / 184$ & 0.59 & 9.3 & 15.0 & 6.5 \\
\hline 19 & $<0.04$ & \multicolumn{2}{|c|}{$1.8_{-0.1}^{+0.1}$} & $0.38_{-0.21}^{+0.21}$ & 9.9 & 0.65 & & $748.87 / 885$ & 1.45 & 1.4 & 4.0 & 8.0 \\
\hline 20 & $12.62_{-7.20}^{+6.96}$ & $1.7_{-1.1}^{+1.0}$ & $1.7_{-1.1}^{+1.0}$ & $0.35_{-0.35}^{+0.51}$ & 9.7 & 0.10 & 0.052 & $140.82 / 167$ & 0.04 & 0.3 & 0.4 & 5.9 \\
\hline 21 & $<0.08$ & \multicolumn{2}{|c|}{$2.2_{-0.2}^{+0.2}$} & * & 4.7 & 1.21 & & $214.46 / 247$ & 0.10 & 0.5 & 0.5 & 6.9 \\
\hline $22^{\dagger}$ & $243.07_{-115.26}^{+303.45}$ & $2.6_{-0.2}^{+0.2}$ & $1.8^{\ddagger}$ & $0.65_{-0.60}^{+0.81}$ & 4.7 & 0.37 & 0.003 & $296.79 / 365$ & 3.07 & 11.7 & 23.3 & 7.8 \\
\hline 23 & $0.39_{-0.22}^{+0.39}$ & \multicolumn{2}{|c|}{$1.5_{-0.4}^{+0.5}$} & $<0.88$ & 16.5 & 0.62 & & $172.56 / 170$ & 0.13 & 0.4 & 1.1 & 7.4 \\
\hline 24 & $6.47_{-1.07}^{+1.31}$ & $2.0_{-0.3}^{+0.4}$ & $2.0_{-0.3}^{+0.4}$ & $0.17_{-0.13}^{+0.17}$ & 41.1 & 0.77 & 0.009 & $659.27 / 707$ & 0.26 & 2.3 & 3.4 & 7.1 \\
\hline $25^{\dagger}$ & $<0.07$ & \multicolumn{2}{|c|}{$0.8_{-0.2}^{+0.2}$} & $<0.70$ & 22.7 & 0.34 & & $274.4 / 258$ & 0.16 & 0.9 & 2.5 & 6.3 \\
\hline 26 & $1.98_{-0.47}^{+0.50}$ & \multicolumn{2}{|c|}{$1.7_{-0.3}^{+0.3}$} & $<0.37$ & 46.5 & 3.47 & & $383.72 / 507$ & 1.48 & 42.3 & 97.8 & 8.1 \\
\hline 27 & $5.29_{-3.53}^{+4.69}$ & \multicolumn{2}{|c|}{$1.6_{-1.1}^{+1.2}$} & $<0.60$ & 13.1 & 0.23 & & $97.74 / 80$ & 0.22 & 1.4 & 1.9 & 7.1 \\
\hline 28 & $28.78_{-12.22}^{+15.82}$ & $1.7_{-0.7}^{+0.5}$ & $1.7_{-0.7}^{+0.5}$ & $<0.46$ & 34.6 & 2.56 & 0.045 & $246.1 / 310$ & 10.66 & 2.4 & 32.3 & 8.8 \\
\hline $29^{\dagger}$ & $32.18_{-12.68}^{+19.90}$ & $3.4_{-0.3}^{+0.4}$ & $1.8^{\ddagger}$ & $1.22_{-0.73}^{+1.89}$ & 10.6 & 0.09 & 0.155 & $339.5 / 354$ & 1.71 & 1.5 & 2.1 & 7.4 \\
\hline $30^{\dagger}$ & $14.95_{-7.28}^{+11.92}$ & $3.6_{-0.4}^{+0.5}$ & $1.8^{\ddagger}$ & $2.08_{-1.24}^{+3.05}$ & 3.8 & 0.03 & 0.280 & $266.06 / 300$ & 0.96 & 2.7 & 4.9 & 7.4 \\
\hline 31 & $2.61_{-0.68}^{+0.89}$ & \multicolumn{2}{|c|}{$1.3_{-0.3}^{+0.4}$} & $<0.25$ & 10.3 & 0.51 & & $355.49 / 417$ & 0.45 & 0.2 & 0.4 & 6.2 \\
\hline
\end{tabular}

Notes. (1) sequence number; (2), obscuring column density in atoms $/ \mathrm{cm}^{2} ;(3),(4)$ power-law photon index of the scattered and the continuum emission, respectively (in the case of a single power-law fit. the value is listed in the middle of the two columns); (5) equivalent width in keV of the $\mathrm{FeK} \alpha$ line at $6.4 \mathrm{keV}$; (6) X-ray flux in erg s $\mathrm{em}^{-1}$; (7) X-ray luminosity in erg s${ }^{-1}$; (8) the ratio of the scattered to the continuum emission normalization; (9) C-statistics and degrees of freedom; (10) reddening-corrected [OIII] luminosity in erg s ${ }^{-1}$, (12) AGN bolometric luminosity computed from the SED; (13) black hole mass computed from the $M_{\mathrm{BH}}-\sigma *$ relation. ${ }^{\star}{ }^{\star}$ Not constrained. ${ }^{(\dagger)}$ See Appendix A for notes on individual objects. $\stackrel{(亠)}{*}$ Fixed $\Gamma_{\text {hard}}$.

obeys the relation

$M_{\lambda}=M_{\lambda 0}\left(\sum_{j=1}^{N_{\mathrm{SSP}}} x_{j}, b_{j, \lambda} r_{\lambda}\right) \otimes G\left(v_{\star}, \sigma_{\star}\right)$.

A detailed description of the STARLIGHT code can be found in the publications of the SEAGal collaboration (Cid-Fernandes 2005, 2007; Mateus 2006; Asari 2007). In Fig. 1 we present two examples of the spectral decomposition results.

After subtracting the stellar background, we use the commercial software PEAKFIT, by Systat Software Inc., to model the emission lines. We analyze separately the red $(\mathrm{H} \alpha, \mathrm{N}[\mathrm{II}]$ and $\mathrm{S}[\mathrm{II}]$ emission lines) and the blue ( $\mathrm{H} \beta$ and [OIII] emission lines) parts of the spectrum. We initially model the emission lines in the blue part, since we are mostly interested in the profile of the $[\mathrm{OIII}] \lambda 5007$ narrow emission line, with which we also try to fit the lines in the blue part and especially the $\mathrm{H} \alpha$. We model the [OIII] line with a mixed Gaussian and Lorentzian profile. The contribution of each profile to the fit is a free parameter. If the same profile can also be applied to the red part of the spectrum, we consider this source as a narrow line AGN and keep it in our sample. If there is still a need for an extra broad 

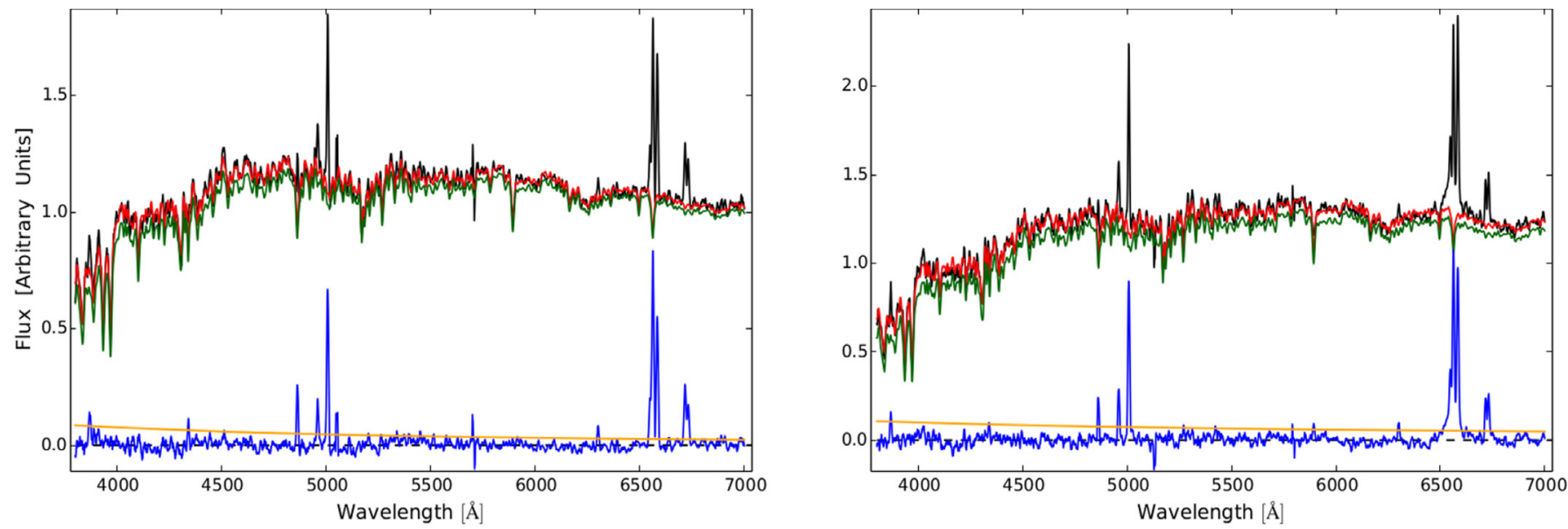

Fig. 1. Two examples of the optical spectrum analysis using STARLIGHT software. We plot the observed spectrum (black), the host galaxy model spectrum (green), the modeled spectrum (red), the AGN continuum emission (yellow), and the residual emission-line-spectrum (blue).

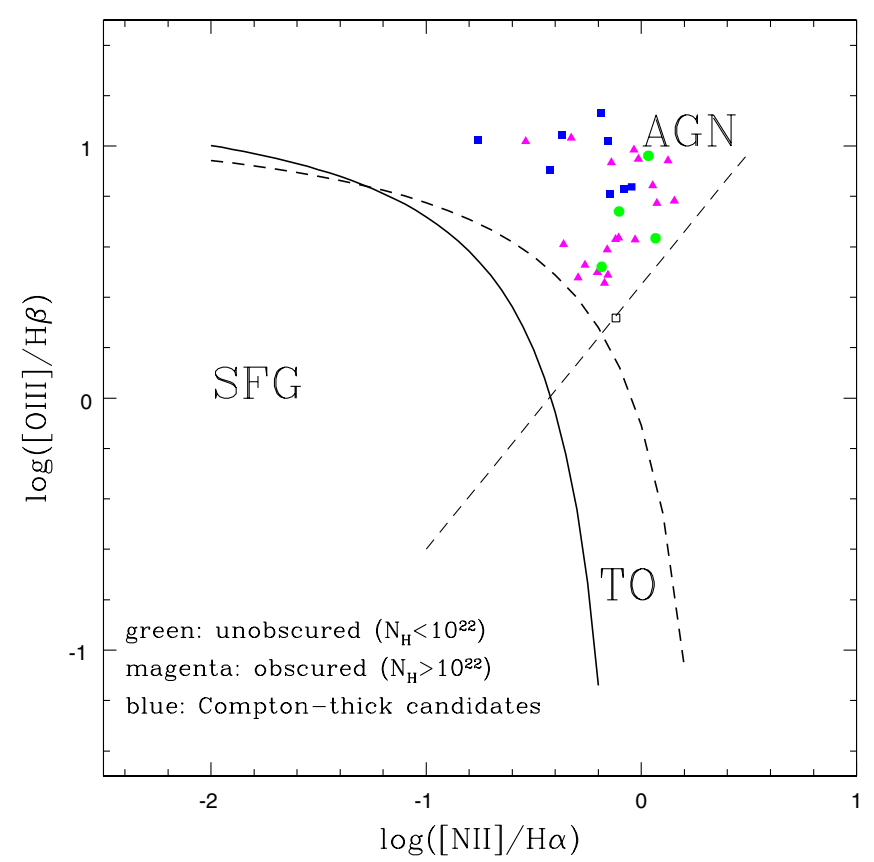

Fig. 2. BPT diagnostic diagram for the Sy2 sample. The various levels of obscuration are color-coded. The continuous curve denotes the star-forming - AGN separation line of Kauffmann et al. (2003), and the thick dashed curve the respective line of Kewley et al. (2001). The dashed straight line denotes the LINER - AGN threshold by Schawinski et al. (2007). Composite or transition objects (TO) between the AGN and star-forming phase are found in the area between the two curves. The open square indicates the position of a discarded LINER.

component to model the $\mathrm{H} \alpha$ the source is discarded. In any case, the $[\mathrm{NII}] \lambda 6583 /[\mathrm{NII}] \lambda 6548$ flux ratio should be $\sim 3$. We find that seven out of the 40 sources present a broad $\mathrm{H} \alpha$ component. Most of these sources belong to the list of ambiguous-type Seyferts that we described in the previous section.

Finally, we plot the BPT diagram anew, this time with the line ratios calculated by the above spectral analysis. Although the differences are small, we find that a source that was already close to the AGN-LINER separating line, falls in the LINER region and is therefore excluded. The BPT diagram is plotted in Fig. 2.

\section{X-ray spectral fitting}

The X-ray data have been obtained with the European Photon Imaging Cameras (EPIC, Strüder et al. 2001; Turner et al. 2001) onboard XMM-Newton. X-ray photons are collected by three detectors (PN, MOS1, and MOS2). All available instrument spectra are modeled simultaneously by using XSPEC, the standard package for X-ray spectral analysis (Arnaud 1996). We used Cash statistics (C-statistics), implemented as cstat in XSPEC to obtain reliable spectral-fitting results even for the lowest quality spectra in our sample. Many of our sources were detected in only one or two of the three detectors (see Table 1).

The X-ray spectra of type 2 AGN are usually complicated and consist of multiple components: power-law, thermal, scattering, reflection, and emission lines (see Turner et al. 1997; Risaliti 2002; Ptak et al. 2006; LaMassa et al. 2009). Therefore, no single model could successfully fit the spectra in all cases. We initially tried to model all spectra with a single absorbed power law, but if the fit was not acceptable we added a second power law. Since a strong line is expected in obscured sources, we fit a Gaussian line for the $\mathrm{FeK} \alpha$ emission line in both cases. In more detail, this includes:

\section{- Single absorbed power law plus Gaussian FeK $\alpha$ line.}

We assumed a standard power-law model with two absorption components (wabs*zwabs*pow in XSPEC notation) to fit the source continuum emission. The first component models the Galactic absorption. Its fixed values are obtained from Dickey \& Lockman (1990) and are listed in Table 1. The second component represents the AGN intrinsic absorption and is left as a free parameter during the modeling procedure. A Gaussian component has also been included to describe the FeK $\alpha$ emission line. We fix the line energy at $6.4 \mathrm{keV}$ in the source rest frame (except in the case of J090036.85+205340.3 (N6) where the line was found at $6.7 \mathrm{keV}$ and implies ionized $\mathrm{Fe}$ ) and the line width $\sigma$ at $0.01 \mathrm{keV}(\sim 10 \%$ of the instrumental line resolution of XMM-Newton). In 12 cases the fitting procedure gives a rejection probability less than 90 per cent and we can accept the model. However, when this simple parametrization is not sufficient to model the whole spectrum, additional components must be included as described in the next paragraph. 
- Double power law plus Gaussian FeK $\alpha$ line.

In the remaining 20 cases, an additional power law was necessary to obtain an acceptable fit (wabs*(pow+zwabs*pow), in XSPEC notation). The additional power law is only absorbed by the galactic column density. Initially, the photon indices of the soft (scattered/unabsorbed) and hard (intrinsic/absorbed) power-law components were tied together. However, in 13 cases the value of the hard power-law photon index $\Gamma_{\text {hard }}$ was too high (the average photon index of the intrinsic power-law measured in AGN is usually 1.8-2), and we needed to untie it from the soft one to obtain an acceptable fit. In the cases where the data quality was not high enough to constrain $\Gamma_{\text {hard }}$, we fixed it to 1.8 (see Table 2).

The X-ray analysis revealed that one of the sources is the brightest galaxy of a contaminating X-ray luminous cluster. We chose to exclude this source from our sample since we cannot provide any reliable X-ray measurements. Our final sample comprises 31 Seyfert 2. In Fig. 3 we present some examples of the $\mathrm{X}$-ray spectra of unobscured $\left(<10^{22} \mathrm{~cm}^{-2}\right.$, left panels $)$ and strong $\mathrm{FeK} \alpha$-line sources (right panels).

\section{Results}

In the next sections we use various criteria and diagnostic diagrams to investigate the possibility that some objects are more obscured than we can infer from their $N_{\mathrm{H}}$ values and that Compton-thick candidate sources are indeed heavily obscured.

\subsection{Candidate Compton-thick sources}

Only two of the sources have $N_{\mathrm{H}}>10^{24} \mathrm{~cm}^{-2}$, consistent with the high values that define Compton-thick sources. Also, sources (N10) and (N12) are consistent with being CT within the uncertainties. However, except for the column density as a direct indicator of obscuration, there are other criteria, based not only on the X-ray but also on the optical and the infrared emission, that could point to possible Compton-thick sources within our sample. In more detail, a heavily obscured source can have one or more of the following characteristics:

1. Flat X-ray spectrum $(\Gamma<1)$. This implies the presence of a strong reflection component that intrinsically flattens the X-ray spectrum at higher energies (e.g., Matt et al. 2000).

2. High equivalent width of the $\mathrm{FeK} \alpha$ line $(\sim 1 \mathrm{keV})$. In this case a Compton-thick nucleus is evident since the line is measured against a heavily obscured continuum (Leahy \& Creighton 1993) or only against the reflected component.

3. Low X-ray to mid-infrared $\left(L_{12}\right)$ luminosity ratio. All Compton-thick sources should have low $L_{2-10} \mathrm{kev}$ to $L_{12}$ ratios, since the mid-IR luminosity of an AGN should be dominated by very hot dust and the X-ray emission should be suppressed by high amounts of absorption (e.g., Lutz et al. 2004; Maiolino et al. 2007).

4. Low X-ray to optical luminosity ratio. The [OIII] line emission originates in the narrow line region and is not affected by the circum-nuclear obscuration. Therefore, the ratio between the observed hard X-ray $(2-10 \mathrm{keV})$ and [OIII] line luminosity could be used as an indicator of the obscuration of the hard X-ray emission (Mulchaey et al. 1994; Heckman et al. 2005; Panessa et al. 2006; Lamastra et al. 2009; LaMassa et al. 2009; Trouille \& Barger 2010).

\subsubsection{Flat X-ray spectrum as an indicator of obscuration}

The first criterion of $\Gamma<1$ is satisfied only by J135436.29+ 051524.5 (N25). However, this source cannot be included in the Compton-thick candidate sources because there is evidence of partial covering. For more detail see the notes on individual objects in Appendix A.

\subsubsection{High equivalent width of the FeK $\alpha$ line as an indicator of obscuration}

The second criterion of a strong FeK $\alpha$ line is satisfied by four objects (see Table 2). Although the presence of the strong line provides robust evidence of their obscuration, all four exhibit lower $N_{\mathrm{H}}$ values than what is expected by a Compton-thick source. Therefore, we also fit these sources with the model of Brightman \& Nandra (2011), which is based on Monte-Carlo simulations. The advantage of this model is that it fits an iron line consistently with the computed $N_{\mathrm{H}}$. Thus, it cannot result in a good fit with a low $N_{\mathrm{H}}$ value and at the same time a high-EW iron line, and vice versa. The fitting confirms that these four sources are indeed Compton-thick. More details can be found in the notes on individual objects in Appendix A. Therefore, we do include them in our list of CT sources.

Also, we need to examine the X-ray spectra of the unobscured sources carefully for the $\mathrm{FeK} \alpha$ line that could give away the presence of obscuration. However, as we can see in Table 2, the line is actually detected only in one out of the five sources, and the equivalent width $(\mathrm{EW})$ is relatively small $\left(0.38_{-0.21}^{+0.21}\right)$. We do not detect the line in the spectra of any other unobscured source, and the given value of the EW is just the upper limit. Thus, there is no evidence of obscuration based on the presence of a FeK $\alpha$ line.

We note that this criterion is not explicit. High equivalent width lines may also appear in the case of anisotropic distribution of the scattering medium (Ghisellini et al. 1991) or in the case of a time lag between the reprocessed and the direct component (e.g., NGC 2992, Weaver et al. 1996). On the other hand, Compton-thick sources with FeK $\alpha$ EW well below $1 \mathrm{keV}$ have been reported (e.g., Awaki et al. 2000, for Mkn1210).

\subsubsection{The $L_{x} / L_{12}$ ratio as an indicator of obscuration}

The detection of a low X-ray to mid-IR luminosity ratio has been widely used as the main instrument for detecting faint Comptonthick AGN, which cannot be easily identified in X-ray wavelengths (e.g., Goulding et al. 2011). This is because the mid-IR luminosity (e.g., $12 \mu \mathrm{m}$ or $6 \mu \mathrm{m}$ ) is a good proxy for the AGN power because it should be dominated by very hot dust that is heated by the AGN (e.g., Lutz et al. 2004; Maiolino et al. 2007). At these wavelengths, the contribution of the stellar light and of colder dust heated by young stars should be small. Gandhi et al. (2009) presented high angular resolution mid-IR $(12 \mu \mathrm{m})$ observations of the nuclei of 42 nearby Seyfert galaxies. These observations provide the least contaminated core fluxes of AGN. These authors find a tight correlation between the near-IR fluxes and the intrinsic X-ray luminosity (the Gandhi relation).

Spitzer observations do not have the spatial resolution to resolve the core, and the infrared luminosity of an AGN is probably contaminated by the stellar background and the star-forming activity of the galaxy. To obtain an estimate of the purely nuclear $12 \mu \mathrm{m}$ infrared luminosity of our sources, we constructed their spectral energy distributions (SED) and computed the various contributions. To model the spectra we used optical data from the 

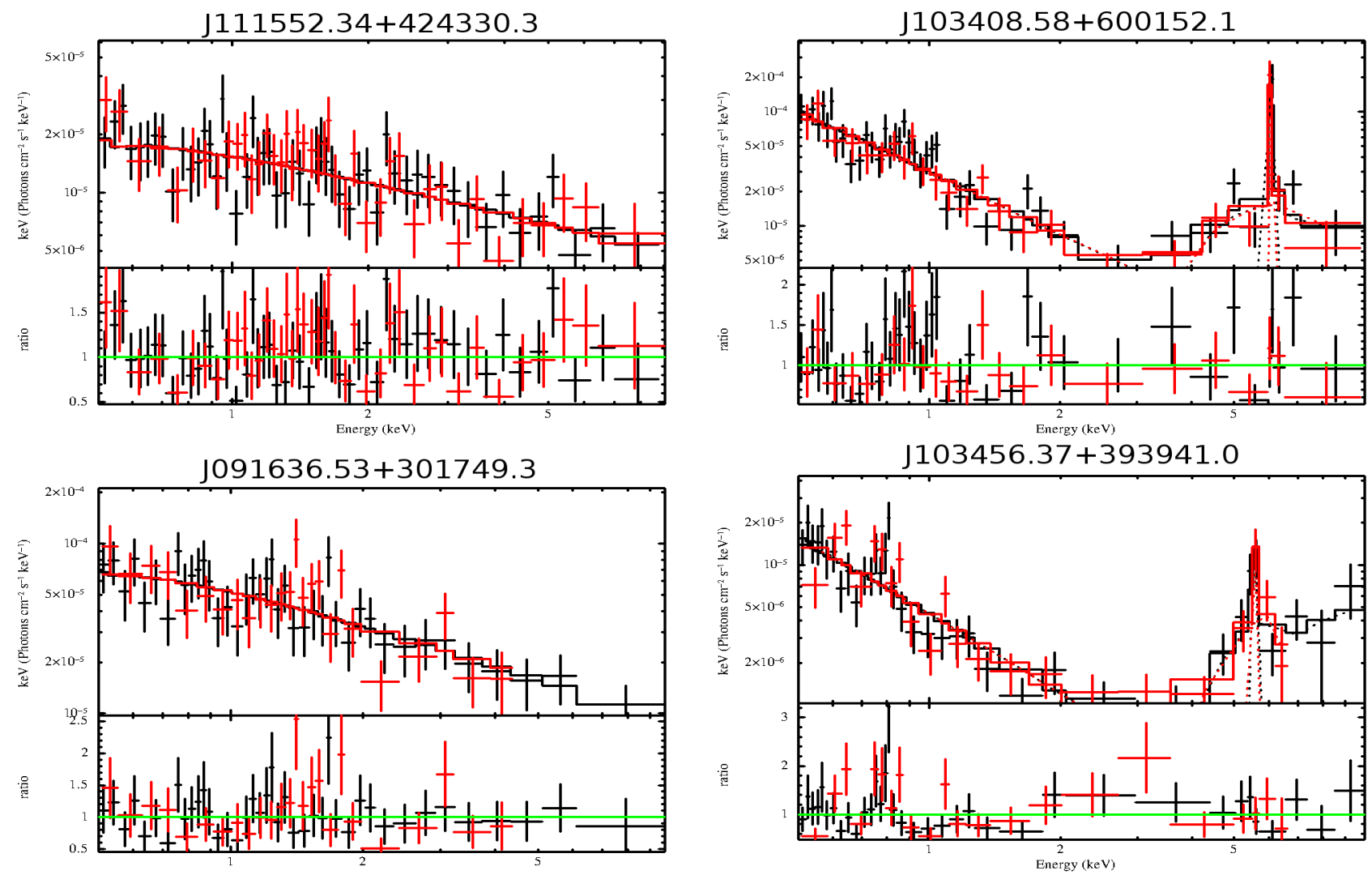

Fig. 3. Left panels: X-ray spectral modeling of two unobscured sources with a single power law. Right panels: X-ray spectral modeling of two candidate Compton-thick sources with a double power law and an iron FeK $\alpha$ line. The black points and the black line denote the PN detector, while the red line denotes the merged MOS1 and MOS2.

SDSS (five optical bands), photometry in the four WISE bands $(3.4,4.6,12$, and $22 \mu \mathrm{m})$ (Wright et al. 2010), and photometry in the three 2 MASS bands $(J, H$, and $K)$ for all sources. Although WISE does include the $12 \mu \mathrm{m}$ band, we are only interested in the AGN contribution, so that the construction of the SED and the decomposition of the AGN and host galaxy component is essential. For more details about the code used, the interested reader should refer to Rovilos et al. (2014, Appendix A).

In Fig. 4 we present the obscured X-ray luminosities against the $12 \mu \mathrm{m}$ luminosities. All our unobscured sources seem to follow the Gandhi-relation closely, and none of them shows unusually high infrared luminosity compared to the X-ray. On the other hand, candidate CT sources are found closer to the dashed line that demarcates the purely $\mathrm{CT}$ region. The sources located below this line are all candidate CT according to our analysis. Therefore, it is is unlikely that we are missing any CT candidates among the Sy2 sample.

\subsubsection{The $N_{H}$ vs. $L_{x} / L[O I I I]$ ratio as an indicator of obscuration}

In this section we investigate the possibility that some of the sources are more obscured than we can infer from their column density.

In Fig. 5 we plot the column density obtained from the $\mathrm{X}$-ray spectral modeling as a function of the X-ray to optical luminosity ratio. The [OIII] luminosities are corrected for reddening using the formula described in Basanni et al. (1999): $\left.\left.L_{[\mathrm{OIII}}\right]_{\mathrm{COR}}=L_{[\mathrm{OIII}}\right]_{\mathrm{OBS}}\left[(\mathrm{H} \alpha / \mathrm{H} \beta) /(\mathrm{H} \alpha / \mathrm{H} \beta)_{\mathrm{o}}\right]^{2.94}$, where the intrinsic Balmer decrement $(\mathrm{H} \alpha / \mathrm{H} \beta)_{\mathrm{o}}$ equals 3. The lower left region
Table 3. Candidate Compton-thick criteria.

\begin{tabular}{lccccc}
\hline \hline$N$ & $\begin{array}{c}\text { Name } \\
(1)\end{array}$ & $\begin{array}{c}N_{\mathrm{H}} \\
(3)\end{array}$ & $\begin{array}{c}\mathrm{FeK} \alpha \\
(4)\end{array}$ & $\begin{array}{c}L_{\mathrm{x}} / L_{12} \\
(5)\end{array}$ & $\begin{array}{c}L_{\mathrm{X}} / L_{[\mathrm{OIII}]} \\
(6)\end{array}$ \\
\hline 9 & $\mathrm{~J} 103408.58+600152.1$ & $>10^{24 \dagger}$ & $\mathrm{x}$ & $\mathrm{x}$ & $\mathrm{x}$ \\
10 & $\mathrm{~J} 103456.37+393941.0$ & $>5 \times 10^{23}$ & & & \\
12 & $\mathrm{~J} 104426.70+063753.8$ & $>9 \times 10^{23}$ & & $\mathrm{x}$ & \\
14 & $\mathrm{~J} 113549.08+565708.2$ & $>10^{24}$ & & $\mathrm{x}$ & $\mathrm{x}$ \\
16 & $\mathrm{~J} 121839.40+470627.6$ & $>10^{24 \dagger}$ & $\mathrm{x}$ & $\mathrm{x}$ & $\mathrm{x}$ \\
22 & $\mathrm{~J} 131104.66+272807.2$ & $>10^{24}$ & & & $\mathrm{x}$ \\
29 & $\mathrm{~J} 150754.38+010816.8$ & $>10^{24 \dagger}$ & $\mathrm{x}$ & & \\
30 & $\mathrm{~J} 215649.51-074532.4$ & $>10^{24 \dagger}$ & $\mathrm{x}$ & $\mathrm{x}$ & $\mathrm{x}$ \\
\hline
\end{tabular}

Notes. (1) sequence number (2); SDSS name, (3) column density $N_{\mathrm{H}}$ in $\mathrm{cm}^{-2}$; (4) sources with a strong $\mathrm{FeK} \alpha$ line; (5) sources located in the CT region of the $L_{\mathrm{x}} / L_{12}$ diagram; (6) sources located out of the $3 \sigma$ region of the $L_{\mathrm{X}} / L_{[\mathrm{OIII}]}$ diagram. ${ }^{(\dagger)}$ The column density of the source is calculated by the Brightman \& Nandra (2011) model (see Appendix A).

in this plot could be possibly occupied by highly obscured or Compton-thick AGN, although their $N_{\mathrm{H}}$ values show the opposite (Akylas \& Georgantopoulos 2009). In our case, however, none of the unobscured sources is located in this region, and therefore there is no evidence that their nuclei are heavily obscured.

On the other hand, three sources with $N_{\mathrm{H}}>10^{23} \mathrm{~cm}^{-2}$ are found marginally outside the $3 \sigma$ limit. This implies that they are probably even more obscured than what we calculated by fitting their X-ray spectra. Interestingly, these are the three out of four sources (J103408.58+600152.1 (N9), 


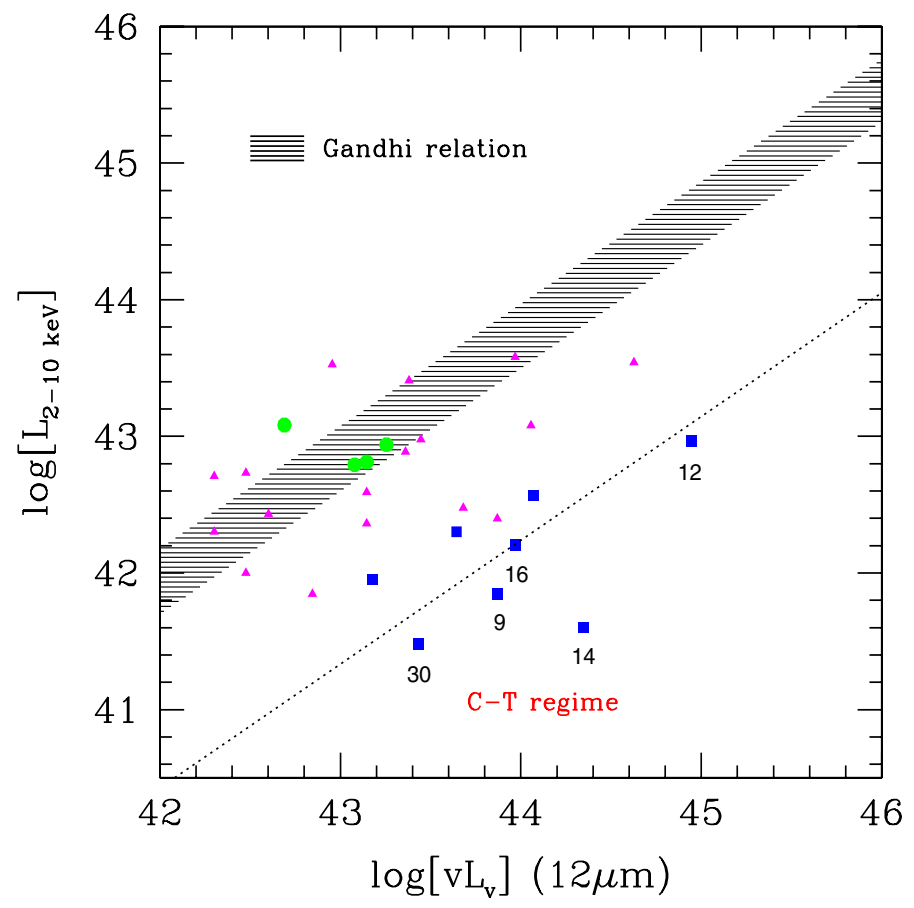

Fig. 4. Absorbed X-ray (2-10 keV) X-ray luminosity against the $12 \mu \mathrm{m}$ luminosity. Color- and shape-coding as in Fig. 1. The typical errors are on the order of $30 \%$ and $20 \%$ for the IR and X-ray luminosity, respectively, including the uncertainties in the model fitting. The hatched diagram represents the $1 \sigma$ envelope of the local (Gandhi et al. 2009) relation. The dotted line corresponds to a factor of 30 lower X-ray luminosity as is typical in many Compton-thick nuclei. The numbers of interesting sources correspond to the sequence numbers in Tables 1 and 2.

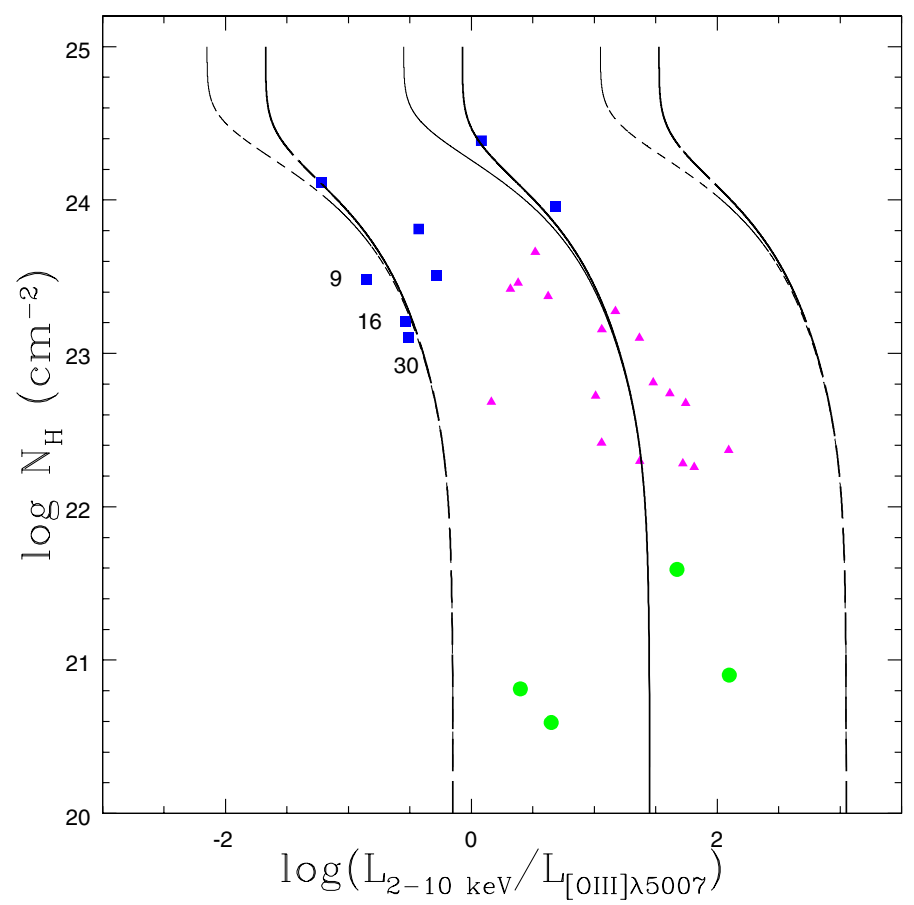

Fig. 5. Distribution of the $N_{\mathrm{H}}$ values as a function of the $L_{2-10 \mathrm{keV}} / L_{[\mathrm{OIII}]}$ ratio. Color- and shape-coding as in Fig. 1. The solid lines represent the mean $N_{\mathrm{H}}$ vs. $L_{2-10 \mathrm{keV}} / L_{[\mathrm{OIII}]}$ relation followed by the Seyfert-1 sample in Akylas \& Georgantopoulos (2009) assuming a photon index of 1.8 and 3\% (thin line: $1 \%$ ) reflected radiation (see also Maiolino et al. 1998; Cappi et al. 2006), while the dashed lines represent the $\pm 3 \sigma$ dispersion. The numbers of interesting sources correspond to the sequence numbers in Tables 1 and 2.
J121839.40+470627.6 (N16), J215649.51-074532.4 (N30)) for which a high $\mathrm{FeK} \alpha \mathrm{EW}$ is reported, and they are also found below the CT line in Fig. 4. Therefore, despite the value of the $N_{\mathrm{H}}$, it is evident that the iron line is a robust indicator of obscuration. Once again we can infer that our classification of unobscured and CT sources is valid.

\section{Discussion and conclusions}

\subsection{Candidate Compton-thick sources}

X-ray spectroscopy shows that the number of Compton-thick AGN in our sample could be as high as eight. N10 was initially included in the CT candidates because it is consistent with being CT within the uncertainties of the calculated column density. However, we chose to exclude this source since it is not confirmed by any of the diagnostics presented in this study (see also LaMassa et al. 2014). Therefore, we are left with seven CT sources, translating to a percentage of $\sim 23 \%$.

We find that the number of CT AGN found in our survey agrees with those in other X-ray surveys of optically selected Seyfert galaxies. In more detail, Akylas \& Georgantopoulos (2009), using XMM-Newton observations, estimate the number of CT sources among the Seyfert galaxies from the Palomar spectroscopic sample of nearby galaxies (Ho et al. 1995). They find a percentage of CT sources of 15-20\%. Since their sample consists of nearby $(<120 \mathrm{Mpc})$ Sy2 galaxies, the X-ray observations provide excellent spectra, hence accurate column density measurements classifications of all the AGN in their sample. Also, Malizia et al. (2009) reports that $\sim 18 \%$ of their hard $\mathrm{X}$-ray selected Sy2 sample is Compton-thick. Nevertheless, considering only the low-redshift sources $(z<0.015)$ to remove the selection bias that affects their sample against the detection of CT objects, the percentage becomes $\sim 35 \%$.

They argue that this result is in excellent agreement with the percentage of CT AGN in the optically selected sample of Risaliti et al. (1999). We note that because of our sample selection, which requires a sufficient number of photons in order to derive X-ray spectra, we may also be biased against heavily obscured sources.

On the other hand, JJ13 in their SDSS optically selected sample of type 2 QSOs, estimate a higher percentage of CT sources that could be as high as $50 \%$, albeit with limited photon statistics. Initially, the percentage they calculate based on the X-ray spectral modeling and the intensity of the $\mathrm{FeK} \alpha$ line is significantly lower. However, it reaches $50 \%$ after they conclude that at least half of the $N_{\mathrm{H}}$ values of their sources are underestimated, based on their $L_{2-10 \mathrm{keV}} / L_{\text {[OIII] }}$ ratios.

Nevertheless, four out of the seven CT sources in our study are in common with JJ13. Three of them are also reported as CT in JJ13. N12 is not a CT source in JJ13 despite its high $N_{\mathrm{H}}$ and the detection of the $\mathrm{FeK} \alpha$ line in their work. A probable reason is that they report an X-ray luminosity that is one order of magnitude higher than the one we measure in the current study. Therefore the $L_{2-10 \mathrm{keV}} / L_{[\mathrm{OIII}]}$ ratio is higher than their threshold for a CT source.

We note that four sources in our sample were initially considered heavily obscured because of the high FeK $\alpha \mathrm{EW}(>1 \mathrm{keV})$, although their column density was only a few times $10^{23} \mathrm{~cm}^{-2}$. This suggests that these sources may be attenuated by CT absorbers. Indeed, all CT sources in the local Universe appear to present high EW of the FeK $\alpha$ line (e.g., Fukazawa et al. 2011) owing to suppression of their continuum emission. The discrepancy between the estimated column density and the EW could 
be attributed to a more complex spectral model that involves a double screen absorber with one of them being CT. In all four cases, by fitting the X-ray spectra with the model of Brightman \& Nandra (2011), we confirm that they are indeed heavily obscured $\left(N_{\mathrm{H}}>10^{24} \mathrm{~cm}^{-2}\right)$. In addition, according to Table 3 , most of them satisfy all our CT criteria. Interestingly, three out of the above four high-EW sources, J103408.58+600152.1 (N9), J215649.51-074532.4 (N30), and J121839.40+470627.6 (N16) lie in the CT regime in the $L_{\mathrm{x}} / L_{12}$ diagram (Fig. 4), and the same three sources have the lowest $L_{\mathrm{X}} / L_{[\mathrm{OIII}]}$ ratios (Fig. 5), again supporting their CT nature. Two of these sources are in common with JJ13 (N9 and N16), and present a high EW in both studies.

\subsection{Unabsorbed Sy2 nuclei}

The X-ray spectral analysis revealed that four ${ }^{3}$ Sy2 galaxies $(\sim 13 \%)$ present very low absorption, below $10^{22} \mathrm{~cm}^{-2}$, in sharp contrast with the unification model of AGN. The percentage of unobscured Sy2 sources varies in the literature, from a few percent $(\sim 3-4 \%)$ in Risality et al. (1999) and in Malizia et al. (2009), to $40 \%$ in Page et al. (2006) and 66\% in Garcet et al. (2007). Our value is in better agreement with Panessa \& Bassani (2002) and Akylas \& Georgantopoulos (2009). However, considering that the number of unobscured Sy2s discovered in any of these studies is less than eight, we argue that we roughly agree with all of them, except perhaps with Garcet et al. (2007). Also, we note that our criteria for selecting narrow line AGN are more stringent than in most of the above studies; for example, Risality et al. (1999) include Sy1.9 in their sample, and Garcet et al. (2007) allow narrow line AGN up to $F W H M_{\mathrm{H} \alpha}=1500 \mathrm{~km} \mathrm{~s}^{-1}$.

As we have already discussed, none of our unobscured sources present a low X-ray to [OIII] or $L_{12}$ luminosity ratio. They also do not present a strong $\mathrm{FeK} \alpha$ line, and therefore we cannot associate them with a highly obscured Compton-thick nucleus. In addition, the FWHM of their $\mathrm{H} \alpha$ line is less than $500 \mathrm{~km} \mathrm{~s}^{-1}$, which excludes the possibility of a narrow-line Sy 1 classification. Although Tran (2001) argues about the presence of this kind of type 2 AGN in his sample of non-HBLR Sy2s fifteen years ago, their existence is still being strongly debated (see discussion in Antonucci 2012). Below, we summarize important observational and theoretical studies in the field, which attempt to approach this problem from various angles.

There is strong evidence that the dusty obscuring torus in low luminosity AGN is absent or is thinner than expected in higher luminosities (e.g., Elitzur \& Shlosman 2006; Perlman et al. 2007; van der Wolk et al. 2010). Accordingly, all low luminosity AGN should have been Type 1 sources, which of course is not the case. The only reasonable explanation of this problem is the additional absence of the BLR in such systems. Some authors (e.g., Nicastro 2000; Nicastro et al. 2003; Bian \& Gu 2007; Marinucci et al. 2012; Elitzur et al. 2014) presented arguments that below a specific accretion rate of material into the black hole, and therefore at lower luminosities, the BLR might also be absent.

Using data from nearby bright AGN, Elitzur \& Ho (2009) conclude that the BLR disappears at bolometric luminosities that are lower than $5 \times 10^{39}\left(M_{\mathrm{BH}} / 10^{7} M_{\odot}\right)^{2 / 3} \mathrm{erg} \mathrm{s}^{-1}$, where $M_{\mathrm{BH}}$ is the mass of the black hole. They also argue that the quenching of the BLR and the disappearance of the torus can occur either simultaneously or in sequence, with decreasing black

\footnotetext{
3 We note that (N25) is a flat X-ray spectrum source with a visible $\mathrm{FeK} \alpha$ line, and we have excluded it from the list of unobscured sources (see Appendix A for more details).
}

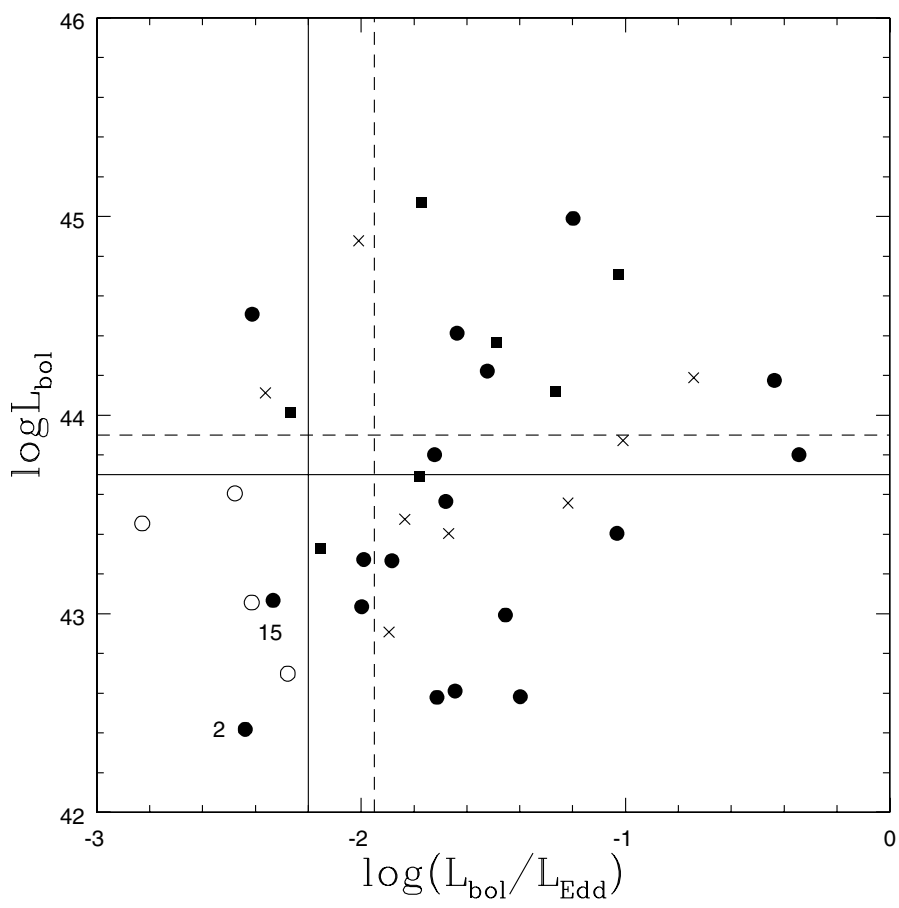

Fig. 6. Eddington ratio vs. bolometric luminosity plot. The open circles denote the unabsorbed Sy2s, and the filled ones the absorbed ones. Squares denote the Compton-thick sources. The solid lines are the empirical thresholds found in the current work below which most of our unobscured Sy2s are found. The dashed lines are the empirical thresholds shown in Marinucci et al. (2012) that separate HBLR and nonHBLR Sy2s.

hole accretion rate and luminosity. Thus, a possible scenario would be that non-HBLR Sy2 AGN are objects lacking the BLR and possibly the torus. Nicastro et al. (2003) conclude that the BLR probably does not exist below an accretion rate threshold of $\log \left(L_{\text {bol }} / L_{\text {Edd }}\right)=-3$, while Marinucci et al. (2012) argue that true Sy2s can be found below the relatively higher limits of bolometric luminosity $\log L_{b o l}=43.9$ and Eddington ratio $\log \left(L_{\text {bol }} / L_{\text {Edd }}\right)=-1.9$. Marinucci et al. (2012) derived the bolometric luminosity from the X-ray and the [OIV] luminosity and conclude that $L_{[\mathrm{OIII}]}$ is not as reliable (see also relevant discussion in Elitzur 2012). We note that Elitzur \& Ho (2009) thresholds are relatively low, not only compared to other studies but also for the general Sy2 population (see discussion in the recent review by Netzer 2015). However, the idea that the accretion rate is essential in the formation of the BLR seems to be valid, although the exact limits have not yet been defined and probably also depend on other factors (see discussion in Koulouridis 2014).

To evaluate the above limits for our four unobscured sources, we computed their bolometric luminosities from the SED modeling (see Sect. 4.2.4). We also calculated their black hole masses using the $M_{\mathrm{BH}}-\sigma *$ relation (Tremaine et al. 2002), where $\sigma *$ is the stellar velocity dispersion, calculated from the FWHM of the [OIII] emission lines (Greene \& Ho 2005). We find that the Elitzur \& Ho (2009) limits are very low for our unobscured sources. Nevertheless, all satisfy the bolometric luminosity and Eddington ratio limit of Marrinucci et al. (2012). We note, however, that our Eddington ratios may be overestimated since the Eddington luminosities, derived from the FWHM of the $[\mathrm{OIII}]$ lines, are probably underestimated (e.g., Bian \& Gu 2007). 
By conducting a two-sample Student's t-test between the accretion rates of the unobscured and the obscured sources, we conclude that their mean values are significantly different at the 99.9\% confidence level. In Fig. 6 we plot the Eddington ratio versus the bolometric luminosity of our Sy2s, but also the discarded intermediate type Seyferts (crosses). We also plot the lines that apparently separate the unobscured sources from the rest of the Sy2 population. These limits are similar to the respective ones found by Marinucci et al. (dashed lines in Fig. 6) for HBLR and non-HBLR sources. All four unobscured sources fall into the area where non-HBLR Sy2s are found and the BLR is predicted to not exist. We note that the limits of previous works were based on the differences between HBLR and non-HBLR Sy2s, while our sample is divided into obscured and unobscured sources. The unobscured Sy2s are non-HBLR Sy2s by definition, whereas the obscured sources are not necessarily HBLR Sy2s. Therefore, the presence of obscured Sy2s in the bottom left quarter of the plot may imply the lack of their BLR as well. Interestingly, a number of Compton-thick sources exhibit low accretion rates. This agrees with the evolutionary scheme of AGN proposed by Koulouridis (2014), where a fraction of Compton-thick sources are predicted to emerge shortly after a galaxy interaction or merging event that causes the inflow of gas and dust toward the central region of the galaxy, enhances circumnuclear star formation and triggers the AGN. During this phase the accretion rate is expected to be low and the BLR absent. However, the failure to detect the BLR in CT sources may as well be due to the heavy obscuration and the large covering factor of the nucleus (see next paragraph). We note that the uncertainties that enter the above calculations are large (see Greene $\&$ Ho 2005) and our samples fairly small. However, the general tendency of low accretion type-2 AGN to lack any evidence of a BLR is once more evident.

An alternative scenario that can explain the lack of detectable BLR in many CT sources is that heavy obscuration does not allow the detection of the BLR even in the polarized spectrum. Marinucci et al. (2012) conclude that $64 \%$ of their comptonthick non-HBLR Sy2s exhibit higher accretion rates than the threshold clearly separating the two Sy2 classes. They attributed this discrepancy to heavy absorption along our line of sight, preventing the detection of the actual BLR in their nuclei. Evidently, merging systems constitute a class of extragalactic objects where heavy obscuration occurs (e.g., Hopkins et al. 2008). The merging process may also lead to rapid black hole growth, giving birth to a heavily absorbed and possibly Compton-thick AGN. Thus, we could presume that a number of our non-HBLR mergers, if not all of them, might actually be BLR AGN galaxies, where the high concentration of gas and dust prohibits even the indirect detection of the broad line emission (e.g., Shu et al. 2007). However, other studies have concluded that there is no evidence that non-HBLR Sy2s are more obscured than their HBLR peers (Tran 2003; Yu \& Hwang 2005; Wu et al. 2011), while totally unobscured low-luminosity non-HBLR Sy2s were detected via investigation of their X-ray properties (e.g., Panessa \& Bassani 2002; Akylas \& Georgantopoulos 2009). The total population of non-HBLR Sy2s is probably a mixture of objects with low accretion rate and/or high obscuration.

Koulouridis (2014) argue that both of the above scenarios agree with an AGN evolutionary scheme (Krongold et al. 2002; Koulouridis et al. 2006a, b, 2013), where a low accretion rate is predicted at the beginning and the end of the Seyfert duty cycle, without ruling out the possibility that some HBLR Sy2s could also be created by minor disturbances or even secular processes.
Finally, we note that there is always the possibility that the discrepancy between the optical and the X-ray spectra is due to variability, since they were not obtained simultaneously.

In a nutshell:

1. We found four unobscured sources $(\sim 13 \%)$ at odds with the simplest unification scheme. These sources exhibit low accretion rates that agree with previous studies that predict the lack of the BLR in low-accretion-rate AGN.

2. $64 \%$ of the Sy2s are obscured with a median column density value of $N_{\mathrm{H}} \sim 1.0 \times 10^{23} \mathrm{~cm}^{-2}$.

3. The percentage of CT AGN is at $\sim 23 \%$, although direct comparison with previous studies is difficult because of the different selection methodologies. Their heavy obscuration was confirmed using a variety of criteria and diagnostics.

Acknowledgements. We thank the anonymous referee for the insightful comments and suggestions that significantly contributed to improving the quality of the publication. E.K. acknowledges fellowship funding provided by the Greek General Secretariat of Research and Technology in the framework of the program Support of Postdoctoral Researchers, PE-1145. This work is based on observations obtained with XMM-Newton, an ESA science mission with instruments and contributions directly funded by ESA Member States and the USA (NASA). Funding for SDSS-III has been provided by the Alfred P. Sloan Foundation, the Participating Institutions, the National Science Foundation, and the US Department of Energy Office of Science. The SDSS-III web site is http://www. sdss3.org/.

\section{References}

Akylas, A., \& Georgantopoulos, I. 2009, A\&A, 500, 999

Akylas, A., Georgakakis, A., Georgantopoulos, I., Brightman, M., \& Nandra, K. 2012, A\&A, 546, A98

Antonucci, R. 1993, ARA\&A, 31, 473

Antonucci, R. 2012, Astron. Astrophys. Trans., 27, 557

Arnaud, K. A. 1996, Astronomical Data Analysis Software and Systems V, 101, 17

Asari, N. V., Cid Fernandes, R., Stasińska, G., et al. 2007, MNRAS, 381, 263

Awaki, H., Ueno, S., Taniguchi, Y., \& Weaver, K. A. 2000, ApJ, 542, 175

Baldwin, J. A., Phillips, M. M., \& Terlevich, R. 1981, PASP, 93, 5

Bassani, L., Dadina, M., Maiolino, R., et al. 1999, ApJS, 121, 473

Bian, W., \& Gu, Q. 2007, ApJ, 657, 159

Brandt, W. N., \& Alexander, D. M. 2015, A\&ARv, 23, 1

Brightman, M., \& Nandra, K. 2011, MNRAS, 414, 3084

Brinchmann, J., Charlot, S., Heckman, T. M., et al. 2004, ArXiv e-prints [arXiv: astro-ph/0406220]

Bruzual, G., \& Charlot, S. 2003, MNRAS, 344, 1000

Cappi, M., Panessa, F., Bassani, L., et al. 2006, A\&A, 446, 459

Cardelli, J. A., Clayton, G. C., \& Mathis, J. S. 1989, ApJ, 345, 245

Churazov, E., Sunyaev, R., Revnivtsev, M., et al. 2007, A\&A, 467, 529

Cid Fernandes, R., Mateus, A., Sodré, L., Stasińska, G., \& Gomes, J. M. 2005, MNRAS, 358, 363

Cid Fernandes, R., Asari, N. V., Sodré, L., et al. 2007, MNRAS, 375, L16

Cid Fernandes, R., Stasińska, G., Mateus, A., \& Vale Asari, N. 2011, MNRAS, 413,1687

Comastri, A. 2004, Supermassive Black Holes in the Distant Universe, 308, 245 Corral, A., Georgantopoulos, I., Watson, M. G., et al. 2015, A\&A, 576, A61

Dickey, J. M., \& Lockman, F. J. 1990, ARA\&A, 28, 215

Dultzin-Hacyan, D., et al. 1999, ApJ, 513, L111

Elitzur, M., \& Ho, L. C. 2009, ApJ, 701, L91

Elitzur, M., \& Shlosman, I. 2006, ApJ, 648, L101

Elitzur, M., Ho, L. C., \& Trump, J. R. 2014, MNRAS, 438, 3340

Frontera, F., Orlandini, M., Landi, R., et al. 2007, ApJ, 666, 86

Fukazawa, Y., Hiragi, K., Mizuno, M., et al. 2011, ApJ, 727, 19

Garcet, O., Gandhi, P., Gosset, E., et al. 2007, A\&A, 474, 473

Georgakakis, A., \& Nandra, K. 2011, MNRAS, 414, 992

Georgantopoulos, I. 2013, Int J. Mod. Phys. Conf. Ser., 23, 1

Georgantopoulos, I., Comastri, A., Vignali, C., et al. 2013, A\&A, 555, A43

Gandhi, P., Horst, H., Smette, A., et al. 2009, A\&A, 502, 457

Ghisellini, G., George, I. M., Fabian, A. C., \& Done, C. 1991, MNRAS, 248, 14

Gilli, R., Comastri, A., \& Hasinger, G. 2007, A\&A, 463, 79

Goulding, A. D., Alexander, D. M., Mullaney, J. R., et al. 2011, MNRAS, 411, 1231 
Greene, J. E., \& Ho, L. C. 2005, ApJ, 627, 721

Heckman, T. M., Ptak, A., Hornschemeier, A., \& Kauffmann, G. 2005, ApJ, 634, 161

Ho, L. C. 2008, ARA\&A, 46, 475

Ho, L. C., Filippenko, A. V., \& Sargent, W. L. 1995, ApJS, 98, 477

Hopkins, P. F., Hernquist, L., Cox, T. J., \& Kereš, D. 2008, ApJS, 175, 356

Hunt, L. K., \& Malkan, M. A. 1999, ApJ, 516, 660

Jia, J., Ptak, A., Heckman, T., \& Zakamska, N. L. 2013, ApJ, 777, 27

Kauffmann, G., Heckman, T. M., Tremonti, C., et al. 2003, MNRAS, 346, 1055

Kewley, L. J., Dopita, M. A., Sutherland, R. S., Heisler, C. A., \& Trevena, J. 2001, ApJ, 556, 121

Koulouridis, E. 2014, A\&A, 570, A72

Koulouridis, E., Plionis, M., Chavushyan, V., et al. 2006a, ApJ, 639, 37

Koulouridis, E., Chavushyan, V., Plionis, M., et al. 2006b, ApJ, 651, 93

Koulouridis, E., Plionis, M., Chavushyan, V., et al. 2013, A\&A, 552, A135

Krongold, Y., Dultzin-Hacyan, D., \& Marziani, P. 2002, ApJ, 572, 169

Krumpe, M., Lamer, G., Corral, A., et al. 2008, A\&A, 483, 415

LaMassa, S. M., Heckman, T. M., Ptak, A., et al. 2009, ApJ, 705, 568

LaMassa, S. M., Yaqoob, T., Ptak, A. F., et al. 2014, ApJ, 787, 61

Lamastra, A., Bianchi, S., Matt, G., et al. 2009, A\&A, 504, 73

Leahy, D. A., \& Creighton, J. 1993, MNRAS, 263, 314

León-Tavares, J., Valtaoja, E., Chavushyan, V. H., et al. 2011, MNRAS, 411, 1127

Levenson, N. A., Weaver, K. A., \& Heckman, T. M. 2001, ApJ, 550, 230

Lutz, D., Maiolino, R., Spoon, H. W. W., \& Moorwood, A. F. M. 2004, A\&A, 418, 465

Maiolino, R., Salvati, M., Bassani, L., et al. 1998, A\&A, 338, 781

Maiolino, R., Shemmer, O., Imanishi, M., et al. 2007, A\&A, 468, 979

Malizia, A., Stephen, J. B., Bassani, L., et al. 2009, MNRAS, 399, 944

Marconi, A., Risaliti, G., Gilli, R., et al. 2004, MNRAS, 351, 169

Marinucci, A., Bianchi, S., Nicastro, F., Matt, G., \& Goulding, A. D. 2012, ApJ, 748,130

Mateus, A., Sodré, L., Cid Fernandes, R., et al. 2006, MNRAS, 370, 721

Matt, G., Fabian, A. C., Guainazzi, M., et al. 2000, MNRAS, 318, 173

Miller, J. S., \& Antonucci, R. R. J. 1983, ApJ, 271, L7

Mulchaey, J. S., Koratkar, A., Ward, M. J., et al. 1994, ApJ, 436, 586

Netzer, H. 2015, ARA\&A, 53, 365

Nicastro, F. 2000, ApJ, 530, L65

Nicastro, F., Martocchia, A., \& Matt, G. 2003, ApJ, 589, L13

Panessa, F., \& Bassani, L. 2002, A\&A, 394, 435

Panessa, F., Bassani, L., Cappi, M., et al. 2006, A\&A, 455, 173

Page, M. J., Loaring, N. S., Dwelly, T., et al. 2006, MNRAS, 369, 156

Pappa, A., Georgantopoulos, I., \& Stewart, G. C. 2000, MNRAS, 314, 589

Perlman, E. S., Mason, R. E., Packham, C., et al. 2007, ApJ, 663, 808

Ptak, A., Zakamska, N. L., Strauss, M. A., et al. 2006, ApJ, 637, 147

Risaliti, G. 2002, A\&A, 386, 379

Risaliti, G., Maiolino, R., \& Salvati, M. 1999, ApJ, 522, 157

Rosen, S. R., Webb, N. A., Watson, M. G., et al. 2015, A\&A, submitted [arXiv: 1504.07051]

Rovilos, E., Georgantopoulos, I., Akylas, A., et al. 2014, MNRAS, 438, 494

Schawinski, K., Thomas, D., Sarzi, M., et al. 2007, MNRAS, 382, 1415

Schlegel, D. J., Finkbeiner, D. P., \& Davis, M. 1998, ApJ, 500, 525

Shu, X. W., Wang, J. X., Jiang, P., Fan, L. L., \& Wang, T. G. 2007, ApJ, 657, 167

Smith, D. A., \& Done, C. 1996, MNRAS, 280, 355

Strüder, L., Briel, U., Dennerl, K., et al. 2001, A\&A, 365, L18

Thomas, D., Steele, O., Maraston, C., et al. 2013, MNRAS, 431, 1383

Tran, H. D. 2001, ApJ, 554, L19

Tran, H. D. 2003, ApJ, 583, 632

Treister, E., Urry, C. M., \& Virani, S. 2009, ApJ, 696, 110

Tremaine, S., Gebhardt, K., Bender, R., et al. 2002, ApJ, 574, 740

Tremonti, C. A., Heckman, T. M., Kauffmann, G., et al. 2004, ApJ, 613, 898

Trouille, L., \& Barger, A. J. 2010, ApJ, 722, 212

Turner, T. J., George, I. M., Nandra, K., \& Mushotzky, R. F. 1997, ApJS, 113, 23

Turner, M. J. L., Abbey, A., Arnaud, M., et al. 2001, A\&A, 365, L27

van der Wolk, G., Barthel, P. D., Peletier, R. F., \& Pel, J. W. 2010, A\&A, 511, A64

Véron-Cetty, M.-P., \& Véron, P. 2010, A\&A, 518, A10

Villarroel, B., \& Korn, A. J. 2014, Nat. Phys., 10, 417

Watson, M. G., Schröder, A. C., Fyfe, D., et al. 2009, A\&A, 493, 339

Weaver, K. A., Nousek, J., Yaqoob, T., et al. 1996, ApJ, 458, 160

Wright, E. L., Eisenhardt, P. R. M., Mainzer, A. K., et al. 2010, AJ, 140, 1868
Wu, Y.-Z., Zhang, E.-P., Liang, Y.-C., Zhang, C.-M., \& Zhao, Y.-H. 2011, ApJ, 730,121

Yu, P.-C., \& Hwang, C.-Y. 2005, ApJ, 631, 720

\section{Appendix A: Notes on individual objects}

- Source 9 - J103408.58+600152.1

Because of the large EW of the FeK $\alpha$ line, but the relatively low $N_{\mathrm{H}}$, we fit the spectrum with the model of Brightman \& Nandra (2011). The result of the fit is a high column density, $N_{\mathrm{H}}=220_{-70}^{+\infty}$, characteristic of the CT sources. Other useful values: $1 / \mathrm{p} 2=0.008$, cstat $/$ d.o.f. $=51.9 / 34, \Gamma_{\text {soft }}=3_{-0.4}^{+0.2}$, $\Gamma_{\text {hard }}=1.8$ (fixed).

- Source 16 - J121839.40+470627.6

Because of the large $\mathrm{EW}$ of the $\mathrm{FeK} \alpha$ line, but the relatively low $N_{\mathrm{H}}$, we fit the spectrum with the model of Brightman \& Nandra (2011). The result of the fit is a high column density, $N_{\mathrm{H}}=2009_{-135}^{+\infty}$, characteristic of the CT sources. Other useful values: $\mathrm{p} 1 / \mathrm{p} 2=0.003$, cstat $/$ d.o.f. $=145 / 155$, $\Gamma_{\text {soft }}=3.4_{-0.6}^{+0.8}, \Gamma_{\text {hard }}=1.8$ (fixed).

- Source 22 - J131104.66+272807.2

Because of the high $N_{\mathrm{H}}$, but the small EW of the FeK $\alpha$ line, we fit the spectrum with the model of Brightman \& Nandra (2011). The result of the fit is a column density value of $N_{\mathrm{H}}=114_{-29}^{+87} \times 10^{22}$, which is relatively lower than what is reported in the current study, but again above the limit that characterize CT sources. A strong FeK $\alpha$ line is only present in the pn detector. Other useful values: p1/p2 $=0.003$, cstat/d.o.f. $=300 / 366, \Gamma_{\text {soft }}=2.6_{-0.2}^{+0.2}, \Gamma_{\text {hard }}=1.8$ (fixed).

- Source 25 - J135436.29+051524.5

We chose not to include this source in the unabsorbed list because its photon index $\Gamma$ is extremely flat $(\sim 0.8)$ if left as a free parameter, and in addition there seems to be a strong $\mathrm{FeK} \alpha$ line. It may be a reflection -dominated Compton-thick source, but we cannot confirm this because of the relatively low quality X-ray spectrum. Also, even though the EW seems high, it cannot be considered as a Compton-thick candidate because the scattered percentage is too large ( $>30 \%)$ implying partial covering instead of scattered emission.

\section{- Source 29 - J150754.38+010816.8}

Because of the large $\mathrm{EW}$ of the $\mathrm{FeK} \alpha$ line, but the relatively low $N_{\mathrm{H}}$, we fit the spectrum with the model of Brightman \& Nandra (2011). The result of the fit is a high column density, $N_{\mathrm{H}}=211_{-61}^{+\infty}$, characteristic of the CT sources. Other useful values: $\mathrm{p} 1 / \mathrm{p} 2=0.003$, cstat $/$ d.o.f. $=320 / 355, \Gamma_{\text {soft }}=3.2_{-0.4}^{+0.4}$, $\Gamma_{\text {hard }}=1.8$ (fixed).

- Source 30 - J215649.51-074532.4

Because of the large EW of the FeK $\alpha$ line, but the relatively low $N_{\mathrm{H}}$, we fit the spectrum with the model of Brightman \& Nandra (2011). The result of the fit is a high column density, $N_{\mathrm{H}}=1500_{-1200}^{+\infty}$, which is characteristic of the CT sources. Other useful values: $\mathrm{p} 1 / \mathrm{p} 2=0.003$, cstat $/$ d.o.f. $=$ $265 / 3, \Gamma_{\text {soft }}=3.5_{-0.5}^{+0.5}, \Gamma_{\text {hard }}=1.8$ (fixed). 